\title{
Naimark, Norman M.: Genozid. Völkermord in der Geschichte, 224 S., Theiss, Darmstadt 2018.
}

\author{
Boris Barth
}

Online publiziert: 12. Mai 2020

(C) Der/die Autor(en) 2020

Norman M. Naimark ist sicherlich einer der profiliertesten internationalen Historiker, die sich mit der Geschichte von Massengewalt in der Neuzeit beschäftigt haben. „Fires of Hatred“ aus dem Jahr 2001 stellt immer noch eine bahnbrechende Pionierstudie zum Thema der ethnischen ,Säuberungen“ im 20. Jahrhundert dar. Bei seiner Definition von Genozid und bei seiner methodischen Herangehensweise folgt Naimark ziemlich genau Raffael Lemkin, der den Terminus 1944 neu geschaffen hat und der maßgeblich an der Schaffung der Genozidkonvention der United Nations Organization (UNO) von 1948 beteiligt war. Lemkins Herangehensweise war dabei nicht unproblematisch: Einerseits wollte er zeigen, dass ,Auschwitz ' eine ganz neue Form von Verbrechen war, andererseits fand er Genozide aber auch in der gesamten Geschichte der Menschheit. Ferner basiert die Konvention der UNO nicht auf einer präzisen wissenschaftlich-historischen Analyse, sondern sie stellt einen politischen Kompromiss zwischen sehr unterschiedlichen Interessen dar. Genau diese Widersprüchlichkeiten finden sich auch in dem neuen Buch von Naimark, der sich der damit verbundenen Problematiken jedoch durchaus bewusst ist.

Naimark unterscheidet zwischen Genoziden in der Antike, Kriegergenoziden, den spanischen Eroberungen, Siedlergenoziden (in Tasmanien, Australien, Nordamerika), modernen Genoziden (darunter die Shoah), kommunistischen Genoziden, antikommunistischen Genoziden (zum Beispiel in Guatemala oder Indonesien mit Osttimor) und Genoziden nach dem Kalten Krieg (in Jugoslawien, Ruanda oder im Kosovo). Eine solche Vorgehensweise ist gewiss legitim, besonders da im angelsächsischen Sprachraum der Begriff genocide meistens eine viel breitere Bedeutung hat als im deutschen. Um einen derartigen Gewaltmarsch durch die Weltgeschichte

\footnotetext{
B. Barth $(\bowtie)$

Prag, Tschechien

E-Mail: boris.barth@uni-konstanz.de

Universität Konstanz, Konstanz, Deutschland
} 
auf circa 200 Seiten bewältigen zu können, ist Naimark aber gezwungen, drastisch zu vereinfachen beziehungsweise Komplexität zu reduzieren und damit auch kleine Ungenauigkeiten in Details in Kauf zu nehmen. Der Preis für dieses Vorgehen ist eine gewisse Unausgewogenheit: Die nationalsozialistischen Völkermorde werden auf etwa 18 Seiten abgehandelt, wobei an der Darstellung insgesamt überhaupt nichts auszusetzen ist. Ungefähr den gleichen Raum nehmen die Folgen der spanischen Eroberungen in der Frühen Neuzeit ein. Hier hätte allerdings viel stärker hervorgehoben werden können, dass die Spanier - trotz aller Grausamkeiten - niemals die Vernichtung der karibischen Indianer geplant oder angestrebt haben. Die eigentlichen Killer, die zur Ausrottung dieser Völker führten, waren Mikroben wie die Masern oder die Pocken, gegen die diese Kariben keine Immunität besaßen.

Bedingt durch diese starke Verdichtung des Stoffes muss Naimark notgedrungen häufig auf komplexere theoretische Erwägungen verzichten, sodass die Darstellung gelegentlich zu einer deskriptiven Aufzählung besonders abscheulicher Grausamkeiten tendiert. Ambivalenzen und Forschungskontroversen bleiben weitgehend auf der Strecke. Dies muss allerdings kein Nachteil sein, weil sich das Buch selbst zumindest in der Sicht des Rezensenten - als eine allgemeine Einführung in das Thema versteht. Die zentrale These des Buches kann und sollte aber doch kritisch diskutiert werden. Naimark nimmt explizit an, dass Genozide seit der Antike ein allgegenwärtiger Bestandteil der menschlichen Zivilisation gewesen seien. Die Genoziddefinitionen des ausgehenden 20. Jahrhunderts würden zu Unrecht den modernen Genozid in den Vordergrund rücken und ließen - im Gegensatz zu Raffael Lemkin - die longue durée unberücksichtigt (S. 194). Die Konsequenz aus dieser sehr weitreichenden These wäre demnach, dass Genozid so etwas wie eine anthropologische Konstante sei, eine Annahme, die der Rezensent bezweifelt.

Das Buch ist in einer sehr flüssigen und gut lesbaren Sprache geschrieben. Es dürfte sich nicht so sehr an den fachlich spezialisierten Historiker, sondern an ein breiteres interessiertes Publikum richten, das mit den jeweiligen Problematiken und Fallstudien weniger vertraut ist.

Funding Open Access funding provided by Projekt DEAL.

Open Access Dieser Artikel wird unter der Creative Commons Namensnennung 4.0 International Lizenz veröffentlicht, welche die Nutzung, Vervielfältigung, Bearbeitung, Verbreitung und Wiedergabe in jeglichem Medium und Format erlaubt, sofern Sie den/die ursprünglichen Autor(en) und die Quelle ordnungsgemäß nennen, einen Link zur Creative Commons Lizenz beifügen und angeben, ob Änderungen vorgenommen wurden.

Die in diesem Artikel enthaltenen Bilder und sonstiges Drittmaterial unterliegen ebenfalls der genannten Creative Commons Lizenz, sofern sich aus der Abbildungslegende nichts anderes ergibt. Sofern das betreffende Material nicht unter der genannten Creative Commons Lizenz steht und die betreffende Handlung nicht nach gesetzlichen Vorschriften erlaubt ist, ist für die oben aufgeführten Weiterverwendungen des Materials die Einwilligung des jeweiligen Rechteinhabers einzuholen.

Weitere Details zur Lizenz entnehmen Sie bitte der Lizenzinformation auf http://creativecommons.org/ licenses/by/4.0/deed.de. 